\title{
Evaluation of the Slip-Spring Dissipative Particle Dynamics Code for Practical Studies in Polymer Rheology
}

\author{
Takeshi AoYAGI ${ }^{\dagger}$ \\ Research Center for Computational Design of Advanced Functional Materials, \\ National Institute of Advanced Industrial Science and Technology (AIST), \\ Central 2, 1-1-1 Umezono, Tsukuba, Ibaraki 305-8568, Japan
}

(Received: November 12, 2020)

\begin{abstract}
We evaluated three slip-spring dissipative particle dynamics models implemented in the general-purpose coarse-grained molecular dynamics program COGNAC. One fixed number of the slip-spring model proposed by Langoloth and co-workers and the two grand canonical models proposed by Unemaya and Masubuchi, and by Ramírez-Hernández and co-workers were implemented based on their original papers. Each implementation was validated by reproducing the original results. The details behind all the settings of the various parameters such as fictitious chemical potentials used in the grand canonical methods were studied. A linear relationship between the slip-spring density and $\exp \left(v / k_{B} T\right)$ was confirmed for the Uneyama and Masubuchi algorithm, where $v$ is a fictitious chemical potential. Moreover, the coefficient for our implementation was determined to enable quantitative adjustments to the slip-spring density for each target application. With the slip-spring model being a promising model in the study of rheological properties of entangled polymer melts and solutions, this work contributes by advancing the application of the slip-spring model both in academia and industry.
\end{abstract}

Key Words: Dissipative particle dynamics / Slip-spring / Molecular simulations / Entanglement

\section{INTRODUCTION}

The concept of polymer entanglement is crucial for studying the viscoelastic properties of polymer melts and solutions. The historical origin of the theoretical study of polymer entanglement is the tube model. ${ }^{1)}$ In its original conception, a single chain is restricted within a tube that mimics the entanglement network and diffuses only along the tube. Following the tube model, a slip-link model was studied. ${ }^{2-7)}$ The slip-link constrains the dynamics of the chain to the transverse plane of the contour of the chain with the chain slipping only along the slip-link. Disentanglement is observed when the end of the chain slips out from the slip-link. Meanwhile, a new slip-link is created at the free end of the chain.

With increases in computational power, more realistic models for entangled polymeric materials were studied. The proposed models considered multichain systems and entanglement between different chains. Doi and Takimoto proposed a multichain slip-link model. ${ }^{4}$ ) This model considered sliplinks that link different chains, from which migration along the chains and construction-destruction of the slip-link were examined. While assuming no spatial distribution of chains, the Doi-Takimoto model randomly selects pairs of chains in the

\footnotetext{
$\dagger^{\dagger}$ Corresponding author.

E-mail: aoyagi.t@aist.go.jp

Tel: +81-29-861-0419
}

system to create the slip-link. Masubuchi and co-workers proposed a primitive chain network model ${ }^{8)}$ this model constructs polymer melts from primitive chains and slip-links connecting the chains in real space.

In both slip-link and primitive chain network models, polymer chains are described as a primitive chains, in which the partial chain between entanglements is highly coarsegrained and only the length or number of Kuhn segments between entanglements are considered.

In addition to the slip-link model, a slip-spring model was proposed as a model with less coarse-graining. ${ }^{9-13)}$ Each chain of the slip-spring model comprises a bead-spring type model, in which coarse-grained beads are connected by harmonic springs, and the slip-spring connects a pair of beads mimicking entanglement.

Dissipative particle dynamics (DPD) is a coarse-grained model for complex fluids and soft matter. ${ }^{14,15)}$ Español and Warren published an excellent review concerning improvements in algorithms and applications of the DPD. ${ }^{15)}$ The DPD employs a soft interaction between beads, allowing chains to pass through each other. Therefore, the original DPD cannot reproduce entanglement effects. Implementing the slip-spring model in DPD provides a practical approach to study rheological properties of homogeneous and heterogeneous melts and solutions of polymers. ${ }^{16-20)}$ 
Although the slip-spring DPD (SS-DPD) is a useful method for studying viscoelastic properties of entangled polymer systems, determining the parameters such as fictitious chemical potentials of slip-spring is non-trivial and requires trial and error fitting for their determination. We implemented the SS-DPD algorithm in the general-purpose coarse-grained molecular dynamics code COGNAC. ${ }^{21)}$ COGNAC is one of simulation engines of OCTA, ${ }^{22,23)}$ which is a multi-scale simulation tool for soft materials and widely used both in academia and industry. We conducted various validations, from which a guide in setting parameters for SS-DPD simulations in regard to specific applications is provided.

\section{SLIP-SPRING MODEL}

The dynamics of the SS-DPD comprises a DPD part and a Monte Carlo (MC) part. A MC trial is executed at every given interval of the DPD time step. The MC step involves three basics steps,

\section{(1) Migration}

Both links at each end of a slip-spring are connected to beads in the polymer chains. Each link migrates to the beads next to the connected bead. The movement is determined based on the spring energy difference of the slip-spring before and after migration.

(2) Destruction

If any links are located at the end of the chain, the link is disentangled and the slip-spring is removed. The criteria for destruction depend on the algorithm.

\section{(3) Construction}

New slip-springs are constructed between the ends of chains and arbitrary beads in any chains nearby. The criteria for this construction also depend on the algorithm.

We implemented the three different slip-spring algorithms in COGNAC. The details of each algorithm are described in the original papers. We reproduced the numerical scheme of the original papers as accurately as possible. In the following, each algorithm are described briefly.

\subsection{Fixed number of entanglement method by Langeloth and co-workers}

Langeloth and co-workers assume a constant number of slip-springs in the model. ${ }^{16)}$ This means that destruction and construction are executed simultaneously. Both destruction and construction of a slip-spring are accepted within the Metropolis criteria of the spring energy difference between destructed slip-spring and constructed slip-spring. This simultaneous action reduces the probability of the total count of destructions and constructions compared with the following algorithms of a grand canonical ensemble.

In addition to the destruction and construction algorithms, the numerical implementation of migration is slightly different for the algorithms. In the original paper, attempts to migrate the two links of one slip-spring are made in one MC step. In this trial migration, with equal probability, one neighboring bead from either side of the original position is selected with the migration of the link of the selected bead being accepted by Metropolis criteria. The number of DPD steps between the $\mathrm{MC}$ trial and the number of MC steps in one trial is adjustable and exploited to reproduce the diffusive property predicted by reptation theory.

Hereafter, we refer to this algorithm as 'model L'.

\subsection{Grand canonical method by Uneyama and Masubuchi}

Uneyama and Masubuchi proposed a grand canonical method in which the number of slip-springs fluctuates based on a fictitious chemical potential $v$ of the slip-spring. ${ }^{9}{ }^{9}$ Masubuchi and co-workers applied this algorithm to DPD model. ${ }^{18)}$ In their algorithm, the movement of the slip-spring is controlled by the friction constant $\zeta_{s}$. Basically, a MC trial is executed at every DPD time step.

The method of migration is as follows. For each MC step, migration is attempted for both links. The probability of migration to either neighbor from an original position is calculated for each link, and the migration is accepted based on the summation of the probabilities. Therefore, the probability of migration at each MC step is larger than that of model L. The selection of the direction is determined by the probability of migration for each direction. Another minor difference is that the criteria of acceptance are based on the Glauber-type dynamics ${ }^{9)}$ and not the Metropolis method.

Hereafter, we refer to this algorithm as 'model U-M'.

\subsection{Grand canonical method Ramírez-Hernández and co-workers}

Ramírez-Hernández and co-workers also proposed a grand canonical approach based on a fictitious chemical potential of the slip-spring $\mu .^{24,25)}$ Although the idea is similar to model U-M, the chemical potentials are not identical because they use different criteria for the destruction and construction of each slip-spring. Following the original papers, we use $\mu$ to denote the chemical potential of the algorithm of RamírezHernández and co-workers, whereas $v$ is used for model U-H. 
Although they did not show the results of the DPD model, it is trivial to implement the algorithm for the DPD. As their method does not introduce a friction constant for the slipspring, the number of DPD steps between a MC trial is an adjustable parameter that is used to control the dynamics of the slip-spring.

Because the criteria regarding destruction and construction consider the spring energy of all bead pairs within the cutoff distance, the calculation cost of one MC step is higher than that for model U-M. Thus, the number of DPD steps between a MC trial should be determined from the perspective of computational cost as well as the reproducibility of the reptation dynamics.

In the original paper, only one link of each slip-spring is selected for migration at any one MC step. Furthermore, occupation of the same bead by more than one link is avoided whereas models L and U-M allow multiple links to the same bead. Thus, the dynamics of the slip-spring are slower than those of the previous two algorithms.

Hereafter, we refer to this algorithm as 'model RH'.

\section{SIMULATION MODEL AND CONDITIONS}

General conditions for the DPD follow those given by Langeloth and co-workers. ${ }^{16)}$ We work in reduced units so that energy $k_{B} T$, mass $m$, and the cutoff length for pair interaction $r_{c}$ are set to unity. Hence, the time unit $\tau$ is $\left(r_{c}^{2} m / k_{B} T\right)^{0.5}$. The number density of beads $\rho$ is set to $3 r_{c}^{-3}$. In addition, a cubic box with periodic boundary conditions is employed. We use typical values for the repulsion parameter between beads $a=25 k_{B} T$ and the spring constant $k=2 k_{B} T / r_{c}{ }^{2}$, for both the polymer bond and slip-spring. Time integration is performed with a time step of $\Delta t=0.06 \tau$ with a modified version of velocity Verlet algorithm. ${ }^{14)}$ The noise amplitude for the thermostat is set to $\sigma=3 k_{B} T \tau^{0.5} / r_{c}$. Since we confirmed that the error of the temperature was the same as that of conventional DPD for all conditions (1.0 percent at largest), no further temperature control was applied.

Although the same DPD conditions is applied to all slipspring models, the number of MC steps for the slip-spring depends on the algorithm. We detail the conditions applying for each slip-spring model.

\subsection{Conditions of model L}

The conditions of model $\mathrm{L}$ were determined from the original paper. ${ }^{16)}$ The number of MC step $N_{M C}$ in one trial was set to 500, as was the number of DPD time step between MC trial $N_{D P D}$. In constructing a new slip-spring, beads within a distance of $0.5 r_{c}$ and $2 r_{c}$ from their selected end bead were extracted as candidates of a pair of beads for the slip-spring.

\subsection{Conditions of model U-M}

The conditions of the model U-M were basically the same as those of the original paper. ${ }^{18)}$ The friction constant of the slip-spring $\zeta_{s}$ was set to 1.0 as a default. $\zeta_{s}$ was set to 3.0 in some cases for a comparison of the dynamic properties. In the construction of a new slip-spring, beads within a distance of $3.0 r_{c}$ were selected.

\subsection{Conditions of model RH}

There is no detailed report of a SS-DPD study for model RH. Therefore, the conditions used in this study were chosen solely to consider the physical accuracy and computational cost. The number of DPD time steps between the MC trial $N_{D P D}$ was set to 20 and beads within a distance of $3.0 r_{c}$ were selected in the construction of a new slip-spring.

\section{RESULTS AND DISCUSSION}

We evaluated our SS-DPD function implemented in COGNAC by comparing the structures and dynamics of the polymer melts, solution, and shear flow of polymer melts of the original works.

\subsection{Homogeneous polymer melts}

The total number of beads for polymer melts was set at 3,000 . The chain length $N$ was set separately to 40,75 , and 100. Because the slip-spring density depends on the fictitious chemical potential in the grand canonical methods, the relationship between the fictitious chemical potential and the slip-spring density must be known to study the rheological properties of the target polymer system.

Figure 1 plots the slip-spring density as a function of $\exp \left(v / k_{B} T\right)$ of model U-M and $\zeta=\exp \left(\mu / k_{B} T\right)$ of model RH. The slip-spring density is proportional to $\exp \left(v / k_{B} T\right)$; see Ref. 9. There is little dependence on chain length as all plots almost overlap. Equation 1 gives the relationship between slip-spring density $\rho_{S S}$ and $\exp \left(v / k_{B} T\right)$ as obtained from linear fitting,

$$
\rho_{S S}=49.3 \exp \left(v / k_{B} T\right) .
$$

By fixing the system density $\rho=3$ used in DPD, we straightforwardly derive the average number of slip-springs in a chain $\langle Z\rangle=2 \rho_{S S} N / 3$ for homopolymer melts of length $N$, 
(a)

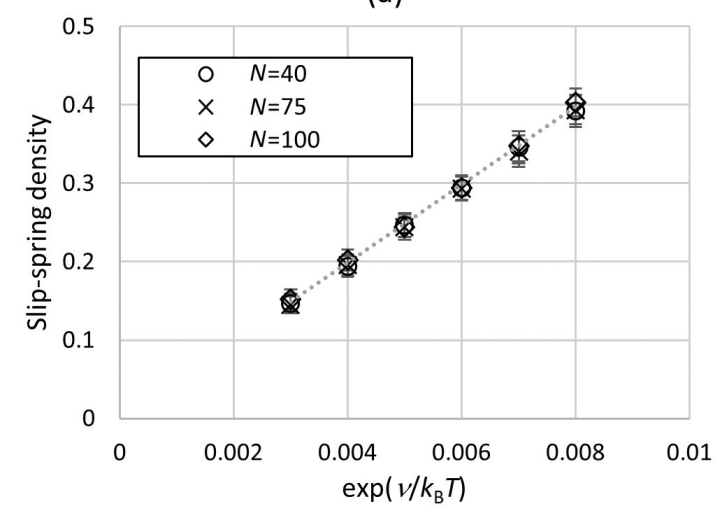

(b)

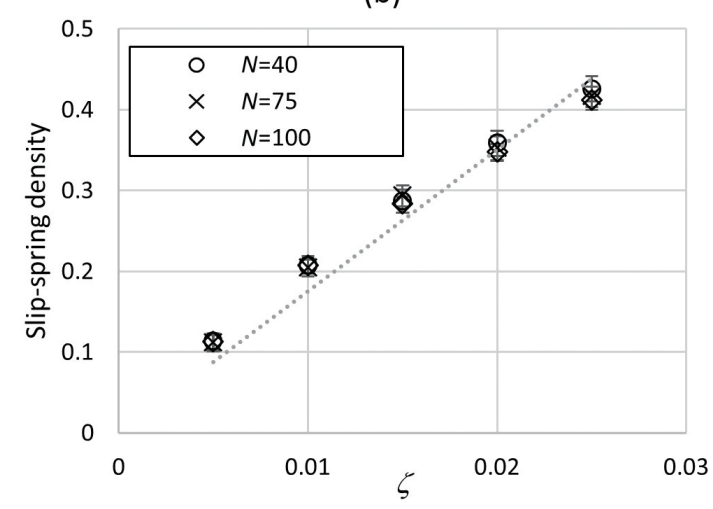

Fig. 1 Slip-spring density as a function of (a) $\exp \left(v / k_{B} T\right)$ for model U-M and (b) $\zeta$ for model RH. The lines are linear fits by proportional function.

and the number of beads between slip-spring $N_{e S S}=3 / 2 \rho_{S S}$. The factor 2 accounts for the fact that both ends of the slipspring connect to polymer chains. This coefficient is valid for our implementation on COGNAC and may depend on the numerical implementation.

Furthermore, for model RH, the slip-spring density deviates slightly from linearity with respect to $\zeta$, as encountered in the original paper. ${ }^{25}$ ) The reason for this discrepancy is as follows. Because a bead occupying two slip-springs is forbidden in model $\mathrm{RH}$, the number of free beads decreases as $\zeta$ increases, as does the slip-spring density.

The study of the structure and dynamics of polymer melts follows the conditions imposed by Langeloth and coworkers. ${ }^{16)}$ The number of slip-springs Nss was set to 300 , which corresponds to $\rho_{S S}=0.3$. The fictitious chemical potentials were determined from their relation (Fig. 1) to reproduce $\rho_{S S}=0.3$. The data points are each an average of three individual runs.

Figure 2 plots the radius of gyration of the three SS-DPD algorithms and the original non-SS DPD. We confirmed that, from the conditions studied, the dynamics of the slip-springs do not perturb the dimension of the polymer.

Figure 3 plots the relaxation modulus $G(t)$ obtained using the three SS-DPD algorithms. The calculation results obtained

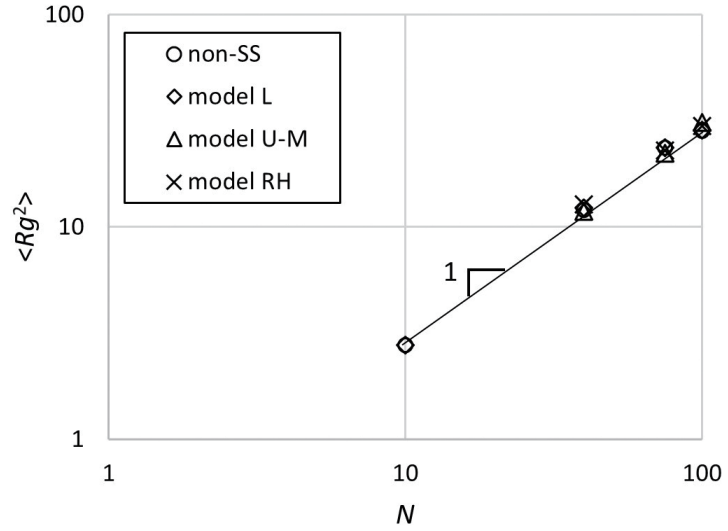

Fig. 2 Radius of gyration obtained from the three SS-DPD algorithms and the non-SS condition.

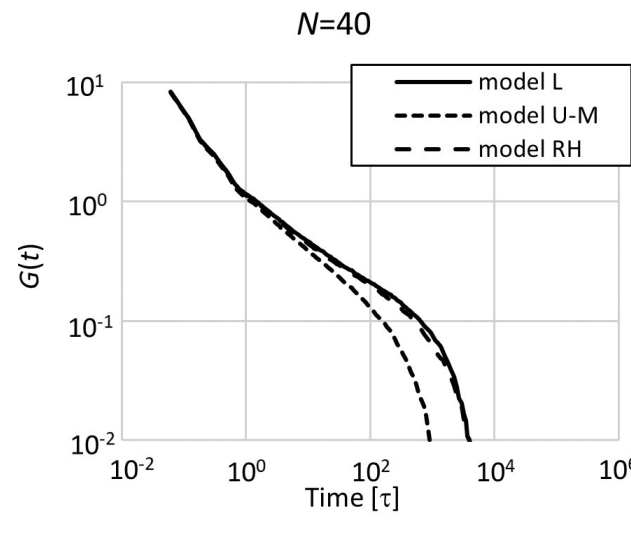

$N=75$

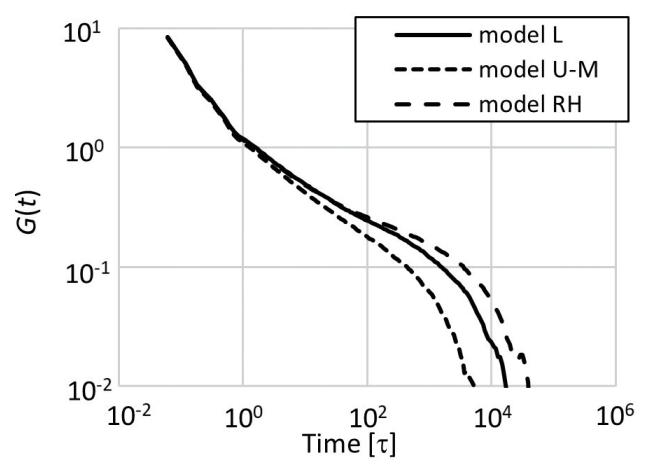

$N=100$

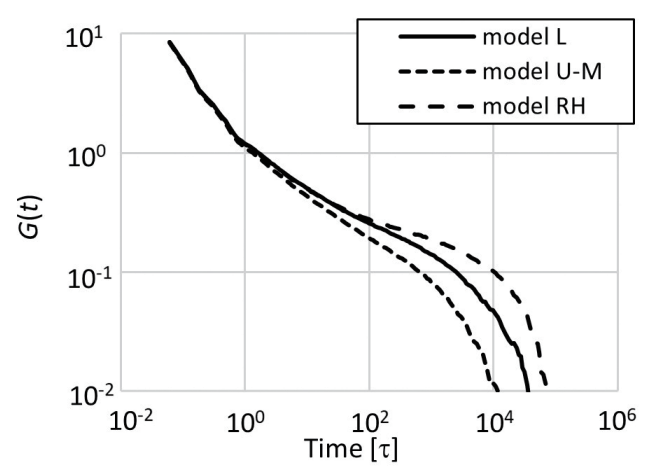

Fig. 3 Relaxation modulus obtained from the three SS-DPD algorithms. 
by our implementation of model $\mathrm{L}$ reproduced the original work shown in Fig. 5 of Ref. 16.

Although the slip-spring densities are the same, stress relaxation of with model U-M is faster than those for the other two models. The migration of the slip-spring is faster than that for the other two algorithms because the criteria applied in the migration process are different, as described above. Therefore, the effects of the constraints for each slip-spring are smaller than for the other algorithms.

As for model RH, the stress relaxation depends on the chain length more than in the other algorithms. The result suggests that this condition constrains the polymer chain more strongly than the other algorithms and the reptation theory. The tendency is confirmed by the mean-squared displacement of the middle beads in chains.

Figure 4 plots the mean square displacement of the middle beads in chains $g_{\text {mid }}(t)$ scaled by $t^{1 / 4}$. The Doi-Edwards theory states that the $g_{\text {mid }}(t)$ of entangled chains shows $t^{1 / 4}$ regime, for which the transverse motion to the chain contour is restricted within the tube, whereas the longitudinal motion is free. The results of model $\mathrm{L}$ shows a clear $t^{1 / 4}$ regime in agreement with the original paper. The results from studies of slip-spring density and constant of friction for model U-M seem to indicate that the constraint on the slip-spring is weaker than that for model L. Conversely, model RH exhibits a strong constraint regime in which the value of $g_{\text {mid }}(t) / t^{1 / 4}$ decreases with time. In this regime, the dynamics longitudinal to the chain contour are somewhat restricted as are the dynamics transverse to it. This may be because in this study $N_{D P D}$ was too large.

We increased the friction constant of model U-M to reduce migration and increase the effect of the constraint of the slip-spring. Figure 5 plots $g_{\text {mid }}(t) / t^{1 / 4}$ for $\zeta_{s}=3.0$. Indeed, the dynamics of the slip-spring slowed down, and an obvious $t^{1 / 4}$ regime is observed. This result suggests that the friction constant as well as the slip-spring density must be determined carefully to correlate the rheological property of the slip-spring model with its experimental measurement. Additionally, when controlling the rheological properties by adjusting the constant of friction, we note that the friction on the slip-spring must be small enough not to perturb the chain dimension.
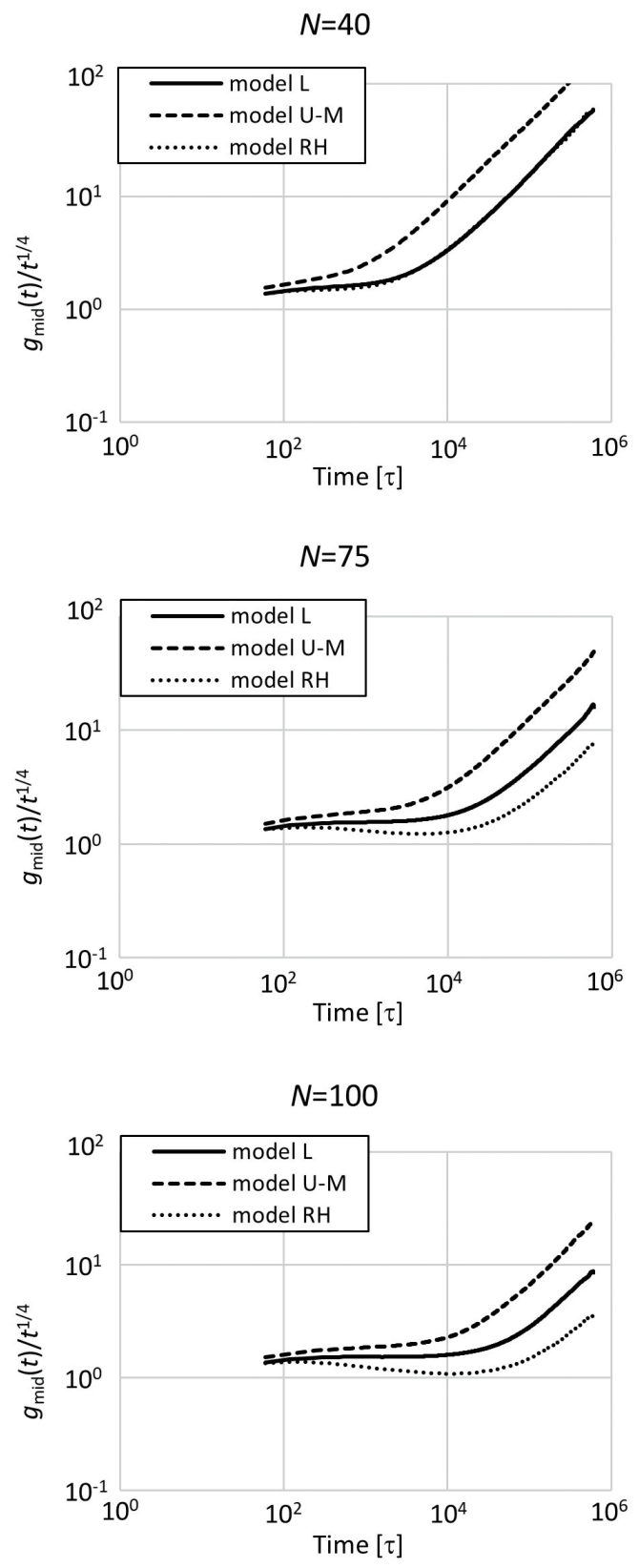

Fig. 4 Mean square displacement of middle beads scaled by $t^{1 / 4}$.

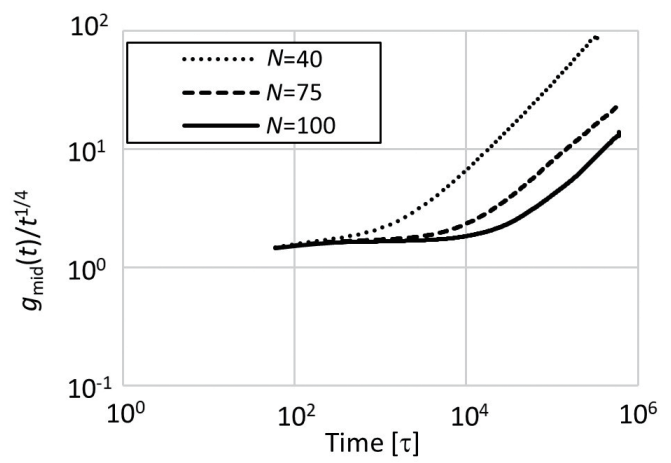

Fig. 5 Mean square displacement of the middle beads scaled by $t^{1 / 4}$ for model $\mathrm{U}-\mathrm{M}$, setting $\zeta_{s}=3$ 


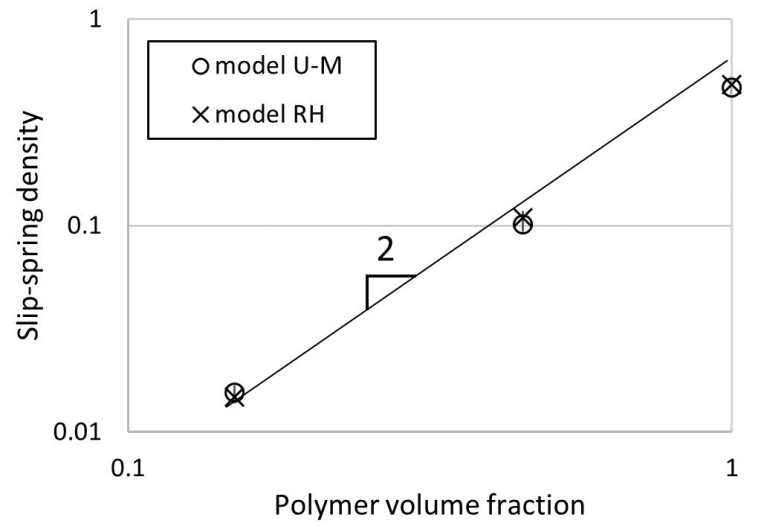

Fig. 6 Slip-spring density as a function of polymer volume fraction.

\subsection{Polymer solution}

The grand canonical algorithm may be applied to a polymer solution, in which the slip-spring density depends on the concentration of the entangled polymer chains. Masubuchi and co-workers studied the rheological properties of a polymer solution using their algorithm SS-DPD. ${ }^{18)}$ We compared the slip-spring density of a polymer solution calculated using models U-M and RH.

For this study of a polymer solution, the total number of beads was set at 7200, and the chain length $N$ was set at 120 . The solvent was a single bead making no link with slip-spring; both polymer chains and solvents were mixed depending on the specified volume fraction of the polymer. The repulsion parameter $a$ between polymer bead and solvent was set to 25 to reproduce a good solvent. The fictitious chemical potential was set so that the average number of slip-springs in a chain $<Z>$ becomes 18.5 in the melt state, as given in the original paper. ${ }^{18)}$

Figure 6 plots the slip-spring density as a function of polymer volume fraction. The results of our implementation of model U-M agreed with the original results (Fig. 5 of Ref. 18). Furthermore, the results of model RH also gave the same results. This result is not surprising. As Masubuchi and co-workers explained in the original paper, ${ }^{18)}$ the slip-spring density must depend on the contact probability between a chain end and another segment, which should be proportional to the square of the polymer volume fraction. This explanation does not depend on the two grand canonical algorithms implemented in this study.

\subsection{Shear flow}

The shear viscosity of polymer melts exhibits non-linear behavior at a shear rate high enough compared with the terminal relaxation time of the system. In such regimes, the slipspring density decreases because of the convective constraint (a)

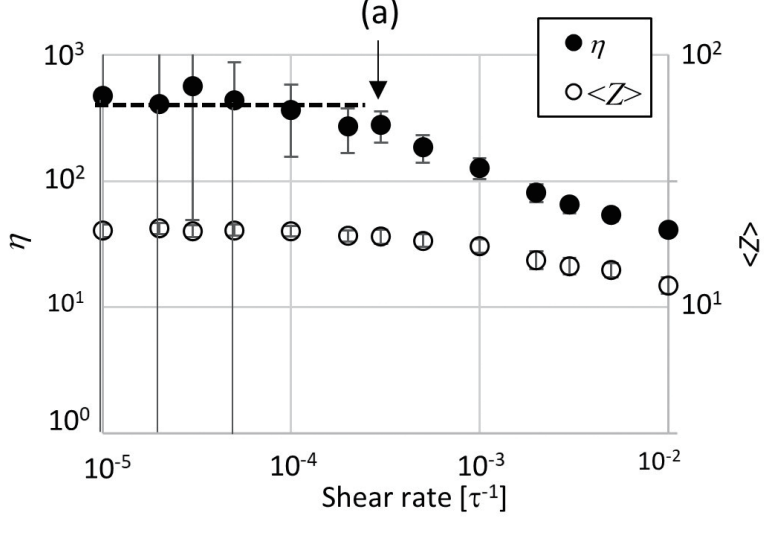

(b)

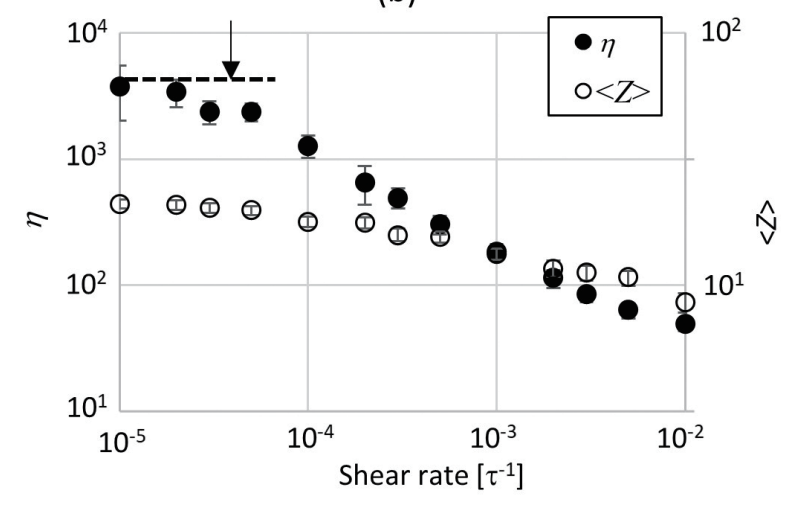

Fig. 7 Shear viscosity $\eta$ and average number of slip-spring $\langle Z\rangle$ in a chain for: (a) model U-M, (b) model RH. The dashed lines identify the zeroshear viscosity calculated from $G(t)$; the arrows mark the estimated transition shear rate from the Newtonian to the non-Newtonian region.

release. ${ }^{26-28)}$ To study this phenomenon, a grand canonical algorithm is used. Ramírez-Hernández and co-workers ${ }^{11)}$ and Masubuchi ${ }^{29)}$ studied the non-linear viscoelasticity of polymer melts under shear flows using a slip-spring model, although they are not DPD-based models. We studied the SS-DPD under shear flows using the two grand canonical algorithms.

Figure 7 plots shear viscosity $\eta$ and $<Z>$ as a function of shear rate obtained by the two algorithms. The system studied contained 3000 beads; the chain length was set to $N=100$, and the initial $\rho_{S S}$ was set to 0.3 , corresponding to $\langle Z\rangle=20$. The Lees-Edwards boundary conditions were applied to the shear flows. Although the deviation increases at a low shear rate, the estimated viscosities were reasonably close to a zeroshear viscosity $\eta_{0}$ calculated using the relaxation modulus $G(t)$ by equation 2 ,

$$
\eta_{0}=\int_{0}^{\infty} G(t) d t .
$$

We note that the stress values of model U-M is about an order of magnitude smaller than those of model RH and the deviation of the viscosity at low shear rate looks very large in 
the plots, even though the actual deviation are the same.

The transition shear rates from the Newtonian to the non-Newtonian regime were roughly estimated as $\dot{\gamma}=$ $3 \times 10^{-4}\left[\tau^{-1}\right]$ and $\dot{\gamma}=4 \times 10^{-5}\left[\tau^{-1}\right]$ for models $\mathrm{U}-\mathrm{M}$ and $\mathrm{RH}$, respectively. The time scales almost correspond to the terminal relaxation time estimated from $G(t)$.

In addition to the difference in time scale, the order of the shear thinning of model RH is slightly larger than that of model U-M. This difference stems from the effect of entanglement of model U-M being weaker than that of model RH.

\section{CONCLUSION}

We implemented three different SS-DPD algorithms into the general-purpose coarse-grained molecular dynamics program COGNAC and conducted detailed validations of each algorithm. The systematic confirmation of the relationship between the fictitious chemical potential and the slip-spring density for the two grand canonical methods offers a guide for the application of this implementation. Furthermore, the friction constant for the slip-spring of model U-M was confirmed to be critical in polymer dynamics and should be studied carefully when comparing results from both other numerical simulations and experiments.

Using implemented three methods, we studied homogeneous polymer melts, polymer solution, and shear flow system. Considering the obtained results, we try to suggest the choice of methods for actual application. Model L is good enough for homogeneous polymer melts without flow, since the slipspring density is constant for the system and the fastest in the three methods. In the case of polymer solution and shear flow system, grand canonical methods should be chosen. Comparing two grand canonical method, model U-M is faster than model RH at the same slip-spring density. However, we note that the entanglement effect depends on the friction constant of slip-spring of model U-M and a care must be taken for determining the parameter.

The original DPD simulation was widely applied in studying multiphase dynamics such as microphase separation of block copolymers and precipitation from polymer solutions. The SS-DPD may be applied to such systems when considering viscoelastic properties based on entanglement. However, the validation of the dynamics of such multiphase systems with entanglement using SS-DPD had not been well studied. COGNAC is an open source program and available from the OCTA web site. ${ }^{23)}$ We are encouraged by the results obtained using COGNAC in our study of SS-DPD models and advocate their further application in similar studies. Model RH is the current choice for such multiphase systems, since model U-M uses mean field density for determining the number of trials for the construction attempt; see Eq. 5 in Ref. 18. This implementation will be improved in future.

Furthermore, various numerical implementations of migration, destruction and construction steps were implemented in this study, and each characteristic was revealed. Moreover, a more effective implementation of the SS-DPD could be possible based on these results in the future study.

\section{ACKNOWLEDGEMENTS}

The author thanks Professor Masubuchi and Professor Uneyama for discussions and especially Professor Masubuchi for providing his code used in the validation. The author also thanks Dr. Tomiyoshi for his original implementation of the slip-spring algorithm in COGNAC. This work was funded by New Energy and Industrial Technology Development Organization of Japan (NEDO) Grant (JPNP16010) and JSPS Grantin-Aid for Scientific Research on Innovative Areas "Discrete Geometric Analysis for Materials Design": Grant Number JP17H06464.

\section{REFERENCES}

1) Doi M, Edwards SF, "The Theory of Polymer Dynamics", (1986), Clarendon Press, Oxford.

2) Hua CC, Schieber JD, Venerus DC, J Chem Phys, 109, 10018 (1998).

3) Rubinstein M, Panyukov S, Macromolecules, 35, 6670 (2002).

4) Doi M, Takimoto J, Phil Trans R Soc Lond A, 361, 641 (2003).

5) Schieber JD, J Chem Phys, 118, 5162 (2003).

6) Likhtman AE, Macromolecules, 38, 6128 (2005).

7) Shanbhag S, Macromolecules, 52, 3092 (2019).

8) Masubuchi Y, Takimoto J, Koyama K, Ianniruberto G, Marrucci G, Greco F, J Chem Phys, 115, 4387 (2001).

9) Uneyama T, Masubuchi Y, J Chem Phys, 137, 154902 (2012).

10) Chappa VC, Morse DC, Zippelius A, Müller M, Phys Rev Lett, 109, 148302 (2012).

11) Ramírez-Hernández A, Müller M, de Pablo JJ, Soft Matter, 9 , 2030 (2013).

12) Vogiatzis GG, Megariotis G, Theodorou DN, Macromolecules, 50, 3004 (2017).

13) Masubuchi Y, Macromolecules, 51, 10184 (2018).

14) Groot RD, Warren PB, J Chem Phys, 107, 4423 (1997).

15) Español P, Warren PB, J Chem Phys, 146, 150901 (2017).

16) Langeloth M, Masubuchi Y, Böhm MC, Müller-Plathe F, J Chem Phys, 138, 104907 (2013).

17) Langeloth M, Masubuchi Y, Böhm MC, Müller-Plathe F, J Chem Phys, 141, 194904 (2014). 
18) Masubuchi $Y$, Langeloth $M$, Böhm MC, Inoue T, Müller-Plathe F, Macromolecules, 49, 9186 (2016).

19) Schneider J, Panagiotopoulos AZ, Müller-Plathe F, J Phys Chem C, 121, 27664 (2017).

20) Schneider J, Süss LD, Müller-Plathe F, J Chem Eng Data, 65 , 1264 (2020).

21) Aoyagi T, Sawa F, Shoji T, Fukunaga H, Takimoto J, Doi M, Comput Phys Commun, 145, 267 (2002).

22) JACI ed, "Computer Simulation of Polymeric Materials. Application of the OCTA System”, (2016), Springer, Singapore.

23) OCTA web site. http://octa.jp/.
24) Ramírez-Hernández A, Peters BL, Andreev M, Schieber JD, de Pablo JJ, J Chem Phys, 143, 243147 (2015).

25) Ramírez-Hernández A, Peters BL, Schneider L, Andreev M, Schieber JD, Müller M, de Pablo JJ, J Chem Phys, 146, 014903 (2017).

26) Marrucci G, J Non-Newtonian Fluid Mech, 62, 279 (1996).

27) Milner ST, McLeish TCB, Likhtman AE, J Rheol, 45, 539 (2001).

28) Ianniruberto G, Marrucci G, J Rheol, 58, 89 (2014).

29) Masubuchi Y, J Chem Phys, 143, 224905 (2015). 